OPEN ACCESS

Edited by:

Nese Celebisoy,

Ege University, Turkey

Reviewed by:

Sohaib Rufai,

Great Ormond Street Hospital for

Children NHS Foundation Trust,

United Kingdom

Kenneth Shindler,

University of Pennsylvania,

United States

*Correspondence:

Heather E. Moss

hemoss@stanford.edu

Specialty section:

This article was submitted to Neuro-Ophthalmology Disorders,

a section of the journa

Frontiers in Ophthalmology

Received: 05 September 2021 Accepted: 04 October 2021

Published: 22 October 2021

Citation:

Aziz R, Shah A and Moss HE (2021) Factors Associated With

Adherence to Outpatient

Follow-Up in Patients With Idiopathic Intracranial Hypertension (IIH).

Front. Ophthalmol. 1:770807. doi: 10.3389/fopht.2021.770807

\section{Factors Associated With Adherence to Outpatient Follow-Up in Patients With Idiopathic Intracranial Hypertension (IIH)}

\author{
Rem Aziz ${ }^{1}$, Asha Shah ${ }^{2}$ and Heather E. Moss ${ }^{3 *}$ \\ ${ }^{1}$ Faculty of Medicine, University of British Columbia, Vancouver, BC, Canada, ${ }^{2}$ Faculty of Medicine, Cambridge Health \\ Alliance, Cambridge, MA, United States, ${ }^{3}$ Departments of Ophthalmology and Neurology \& Neurological Sciences, Stanford \\ University, Palo Alto, CA, United States
}

Idiopathic intracranial hypertension $(\mathrm{IIH})$ is a chronic condition characterized by raised intracranial pressure of undetectable origin, that causes morbidity due to debilitating headaches and vision loss. Continuity of outpatient care is important to monitor for permanent vision loss, manage symptoms and limit emergency care. The purpose of this retrospective study was to identify factors associated with neuro-ophthalmology follow-up appointment completion among patients with $\mathrm{IH}$ at a US academic medical center in order to establish evidence-based interventions to improve adherence patterns. Included are 111 completed or no-show neuro-ophthalmology return outpatient appointments by 23 subjects with $\mathrm{IH}$. Generalized estimating equation models were used to assess association between appointment completion status and factors previously shown to be associated with appointment adherence. Appointments were more likely to be completed during the summer $(p=0.08)$ and by subjects with headache symptoms $(p=0.06)$, however none of the patient factors reached statistical significance. Completed and no-show appointments did not differ by subject demographic or insurance factors. Further studies are needed to identify risk factors for lack of appointment adherence by patients with $\mathrm{IH}$, particularly those amenable to intervention, in order to improve continuity of care for $\mathrm{IH}$.

Keywords: idiopathic intracranial hypertension, outpatient care, resource utilization, follow-up compliance, sociodemographic factors, appointment adherence

\section{INTRODUCTION}

Idiopathic intracranial hypertension (IIH) is a disorder of increased intracranial pressure (ICP), classically occurring in overweight women of childbearing age (1). As per its name, the cause of IIH is unknown, and diagnosis requires confirmation of lack of secondary causes for high ICP such as brain tumor, cerebral venous sinus thrombosis and meningitis. Heterogeneity of IIH is seen with a wide range of visual outcomes including disease progression in some patients despite treatment 
compliance, thus necessitating escalation of therapy and surgical intervention in some cases (2-4). Furthermore, IIH is a chronic condition that can worsen following periods of stability, thereby warranting longitudinal follow-up to optimize outcomes (5). This highlights the importance of ongoing longitudinal followup in preventing progression and sudden deterioration in visual outcomes.

Successful longitudinal care requires adherence to ongoing outpatient appointments and the ability to predictably identify those at risk of being lost to follow up. To establish evidencebased interventions that improve follow-up adherence, it is necessary to first identify factors associated with it. A host of internal and external factors influence adherence behavior, and thus shape health outcomes in return. These can be organized into five major domains: socioeconomic factors, health systemrelated factors, therapy-related factors, condition-related factors, and finally, patient-related factors (6). Accordingly, the goal of the present study was to identify factors associated with completion of recommended outpatient follow-up appointments by IIH patients.

\section{METHODS}

This is a retrospective study of adult patients with IIH followed by an urban academic neuro-ophthalmology service in the University of Illinois Hospital \& Health Sciences System (UIH). This project was reviewed by the University of Illinois at Chicago (UIC) institutional review board under expedited procedures with a waiver of informed consent and waiver of HIPAA authorization.

Subjects were identified by chart review. Adult subjects $(\geq 21$ years old) with confirmed IIH diagnosis, who completed at least one outpatient neuro-ophthalmology appointment between June to September 2014, were included. For these subjects, all neuroophthalmology follow-up encounters between 2012-2015 were abstracted. Canceled, rescheduled, and patient-initiated encounters were excluded. The reason for these exclusions was to focus on patient adherence to provider recommended followup. The included encounters were classified as completed or no show.

Subject demographic factors including age, race/ethnicity, zip code, insurance status (private or government) were abstracted from the medical record. Residential distance from the clinic was calculated through Google Maps, using the subject's zip code to measure the shortest driving distance from home to UIH. Health status was determined based on prescription of medications for diseases other than IIH.

Appointment factors were abstracted from the electronic scheduling system and medical record, and included: time of day (morning or afternoon), season (winter, spring, summer, fall), follow-up time from last completed appointment (1 week, 1 month, 2 months, 3 months, 6 months), whether subject is receiving treatment for $\mathrm{IIH}$, IIH medication change at or since prior appointment, and symptoms at last appointment (headache or not).
Appointment status was the primary outcome. Generalized Estimating Equation (GEE) analysis was applied to model this as a dichotomous outcome (no show vs. completed) using logit link with subject as a grouping variable to account for within subject correlations. Unadjusted analysis was performed for each considered subject and appointment factor. Analysis was performed using SPSS 26 (IBM Inc).

\section{RESULTS}

Twenty-three subjects with IIH (mean age 39, all female, 14 (60\%) Black non-Hispanic, 0-66 miles between residence and clinic, 19 (82\%) on IIH medication, 11 (47\%) with Medicaid insurance) had 321 appointments scheduled between 2012-2015. Two hundred and ten were excluded for being cancelled, rescheduled, initial consultation, patient-initiated, visual field only or undocumented appointments. The remaining 111 appointments were categorized as completed $(n=92,83 \%$, range $1-12$ per patient) or no-show $(n=19,17 \%$, range $0-5$ per patient) visits.

None of the patient factors were associated with adherence to outpatient appointments. Appointments scheduled in the summer season had higher adherence, and those with 1 month follow-up interval had lower adherence, but these did not reach statistical significance. Subjects with active headache had better follow-up, but this did not reach statistical significance. There was no association between other appointment factors (time of day, season, follow-up time, treatment status, mediation changes, symptoms reported) and outpatient appointment adherence among IIH patients (Table 1).

\section{DISCUSSION}

The understanding of factors associated with continuity of outpatient care for those with IIH is important for designing interventions to improve outcomes in this chronic disease. Secondary benefits would include more cost-effective care and reduced overall resource utilization (7). Towards this end, we present a study assessing factors related to outpatient follow-up adherence in patients with $\mathrm{IIH}$, receiving care at an urban academic medical center in the United States.

Our study of established IIH patients in a neuroophthalmology practice did not identify intervenable factors associated with outpatient follow-up completion. Trends for seasonal effects existed, with compliance being better in the summer than the winter, possibly attributable to the weather in Chicago or fewer work/school scheduling conflicts. Follow-up completion was better in subjects with persistent symptoms. However, neither of these factors reached statistical significance, perhaps related to sample size. Ongoing study is critical given the importance of outpatient follow-up to allow management of chronic conditions. In other diseases correlations between outpatient adherence and cultural, sociodemographic and psychosocial factors have been demonstrated $(8,9)$. 
TABLE 1 | Distribution of patient and appointment factors by attendance, and association with non-adherence.

\begin{tabular}{|c|c|c|c|c|}
\hline Factors & $\%$ No-show $(n=19)$ & $\%$ Completed $(\mathbf{n}=92)$ & Odds Ratio $(95 \% \mathrm{Cl})^{\star}$ & P-value* \\
\hline Age in years (Mean $\pm S D$ ) & $41.7 \pm 8.6$ & $43.0 \pm 10.6$ & $0.99(0.94-1.04)$ & 0.64 \\
\hline Race/ethnicity: & & & $1.48(0.43-5.10)$ & 0.53 \\
\hline Black-non-Hispanic & $63 \%$ & $72 \%$ & & \\
\hline Distance from clinic (miles) & $0-66$ & $0-23$ & $1.032(0.98-1.09)$ & 0.28 \\
\hline Insurance status: Public aid & $26.3 \%$ & $32.6 \%$ & $1.36(0.61-3.00)$ & 0.46 \\
\hline Health status: Receiving meds & $94.7 \%$ & $84.6 \%$ & $0.31(0.06-1.65)$ & 0.17 \\
\hline Time of day: AM & $68 \%$ & $66 \%$ & $0.91(0.32-2.55)$ & 0.89 \\
\hline \multicolumn{5}{|l|}{ Season } \\
\hline Spring & $26 \%$ & $19 \%$ & $1.14(0.32-4.02)$ & 0.84 \\
\hline Summer & $16 \%$ & $33 \%$ & $3.16(0.86-11.60)$ & 0.08 \\
\hline Fall & $26 \%$ & $27 \%$ & $1.58(0.49-5.06)$ & 0.44 \\
\hline Winter & $32 \%$ & $21 \%$ & ref & \\
\hline \multicolumn{5}{|l|}{ Follow-up time } \\
\hline 1-2 weeks & $5 \%$ & $12 \%$ & ref & 0.06 \\
\hline 1 month & $42 \%$ & $25 \%$ & $0.26(0.06-1.08)$ & 0.40 \\
\hline 2 months & $11 \%$ & $10 \%$ & $0.41(0.05-3.23)$ & 0.73 \\
\hline 3-4 months & $21 \%$ & $35 \%$ & $0.73(0.12-4.55)$ & 0.33 \\
\hline 6 months & $21 \%$ & $18 \%$ & $0.39(0.06-2.62)$ & \\
\hline IIH treatment: Yes & $79 \%$ & $72 \%$ & $0.68(0.18-2.61)$ & 0.57 \\
\hline Recent IIH med change: Yes & $47 \%$ & $39 \%$ & $0.71(0.38-1.51)$ & 0.38 \\
\hline IIH symptoms (headache): Yes & $53 \%$ & $76 \%$ & $2.82(0.96-8.30)$ & 0.06 \\
\hline
\end{tabular}

*Odds ratio and p-values calculated using GEE models accounting for within subject correlation.

Further research is needed to identify patient-related and health-system factors that are associated with follow-up care in IIH so that patients at risk of poor follow-up can be identified and appropriately managed. As an example of what might be accomplished, one study evaluating follow-up patterns in HIVseropositive patients led to proposed interventions to minimize sociodemographic barriers to appointment adherence in order to promote optimal management of disease progression (8). Similarly, literature surrounding diabetes follow-up asserts the influence of psychosocial factors in chronic disease management, highlighting that patients with attachment styles characterized by low levels of collaboration were more likely to miss appointments than those with secure attachment styles (10). It is worth noting that many of those with poor follow-up compliance will naturally be the most challenging to access during observational research studies. Thus, it is important to critically evaluate solutions drawn from studies that focus on more accessible populations, and contextualize them in terms of those most lost to care $(11,12)$. Future research regarding follow-up disparities in IIH patients will need to be more encompassing of underrepresented populations with greater likelihood of experiencing the greatest health needs.

Intentional versus unintentional nonadherence would be another notable avenue to assess as it has shown importance in research concerning compliance of glaucoma patients with ocular hypotensive therapies (13). Additionally, identifying factors affecting adherence in patients from different genders and cultural backgrounds may be significant to understanding follow-up patterns in patients with IIH, majority of whom are females. In a recent study, beliefs about glaucoma treatment were predictive of adherence in patients of western cultures, suggesting possible improvement of adherence patterns through examination of patient concerns and illness perceptions (14). Finally, a thorough assessment of tailored interventions and their impact on medical adherence and health outcomes would strengthen their implementation to help patients with IIH achieve better follow-up.

Our study is based on the assumption that longer follow-up leads to better health outcomes. Though this has not been formally evaluated in patients with $\mathrm{IIH}$, a recent prospective study demonstrated an association between changes in IIH care prompted by the COVID-19 lockdown and changes in management (15). Moreover, established outpatient care has been associated with better outcomes in other chronic disease conditions. In a study of outpatient diabetes management, consistent appointment attendance was a strong predictor of successful disease control (16). Similarly, a case study examining attendance at outpatient clinics identified appointment nonadherence as a significant disadvantage in the provision of effective medical care (17). A literature review looking at nonadherence in chronic disease conditions identified numerous consequences in medical care provision impacting individual patients and overall health systems (18). These included increased wait times, strained patient-provider relationships, and increased system costs in addition to accumulative negative impacts on patient health, which limit continuity of care and disrupt management plans (19-21). Such conditions delay necessary treatment, leaving patients at an increased vulnerability for the development of serious medical complications. Rigorous study is needed to demonstrate the importance of outpatient specialty care in improving IIH outcomes while reducing costs.

Our findings are not without limitations. Generalizability of our study is limited by its retrospective nature and restriction to a single center, thereby excluding geographic variability and possible relevant care obtained from outside providers. We had a small sample size which was driven in part by restriction to subjects with a confirmed IIH diagnosis only. Future work could include a larger sample size to reveal statistically significant 
associations and enable subgroup analyses. We were unable to capture cultural, sociodemographic, and psychosocial factors, which as discussed earlier, pose important considerations for adherence and follow-up. Future studies may consider directly exploring reasons for poor adherence through the use of patient questionnaires or interviews. Additionally, it would be worthwhile to revisit patients with cancelled and rescheduled appointments, who were originally excluded in our inclusion criteria, to assess whether adherence to follow-up care took place.

In conclusion, our pilot study did not identify specific factors upon which to intervene to establish outpatient care and maintain appointment compliance. Thus, further research is required to better understand factors associated with continuity of care for patients with IIH in order to facilitate a shift to longitudinal outpatient care. Ultimately, this would serve the purpose of continued follow-up for optimal management of $\mathrm{IIH}$, as well as the potential to minimize IIH-related healthcare costs. One particularly interesting approach would be to map out the sequence and stages of care in patients with $\mathrm{IIH}$, such as diagnosis, management and maintenance, in order to identify patterns, their possible causes and appropriate interventions to implement.

\section{DATA AVAILABILITY STATEMENT}

The raw data supporting the conclusions of this article will be made available by the authors, without undue reservation.

\section{REFERENCES}

1. Wall, M. Idiopathic Intracranial Hypertension. Neurol Clin (2010) 28(3):593617. doi: $10.1016 /$ j.ncl.2010.03.003

2. Wall, M, Falardeau, J, Fletcher, WA, Granadier, RJ, Lam, BL, Longmuir, RA, et al. Risk Factors for Poor Visual Outcome in Patients With Idiopathic Intracranial Hypertension. Neurology (2015) 85(9):799-805. doi: 10.1212/ WNL.0000000000001896

3. Corbett, JJ, Savino, PJ, Thompson, HS, Kansu, T, Schatz, NJ, Orr, LS, et al. Visual Loss in Pseudotumor Cerebri. Follow-Up of 57 Patients From Five to 41 Years and a Profile of 14 Patients With Permanent Severe Visual Loss. Arch Neurol (1982) 39(8):461-74. doi: 10.1001/archneur.1982.00510200003001

4. Orcutt, JC, Page, NGR, and Sanders, MD. Factors Affecting Visual Loss in Benign Intracranial Hypertension. Ophthalmology (1984) 91(11):1303-12. doi: 10.1016/S0161-6420(84)34149-5

5. Shah, VA, Kardon, RH, Lee, AG, Corbett, JJ, and Wall, M. Long-Term Follow-Up of Idiopathic Intracranial Hypertension. Neurology (2008) 70 (8):634-40. doi: 10.1212/01.wnl.0000299893.43918.a8

6. World Health Organization. Adherence to Long-Term Therapies: Evidence for Action. Geneva, Switzerland: WHO, Marketing and Dissemination (2003). 1-199.

7. Koerner, JC, and Friedman, DI. Inpatient and Emergency Service Utilization in Patients With Idiopathic Intracranial Hypertension. J Neuro-Ophthalmol (2014) 34(3):229-32. doi: 10.1097/WNO.0000000000000073

8. Israelski, D, Gore-Felton, C, Power, R, Wood, MJ, and Koopman, C. Sociodemographic Characteristics Associated With Medical Appointment Adherence Among HIV-Seropositive Patients Seeking Treatment in a County Outpatient Facility. Prev Med (Baltim) (2001) 33:470-5. doi: 10.1006/ pmed.2001.0917

9. Apter, AJ, Reisine, ST, Affleck, G, Barrows, E, and ZuWallack, RL. Adherence With Twice-Daily Dosing of Inhaled Steroids: Socioeconomic and Health-

\section{ETHICS STATEMENT}

The studies involving human participants were reviewed and approved by University of Illinois at Chicago (UIC) institutional review board. Written informed consent for participation was not required for this study in accordance with the national legislation and the institutional requirements.

\section{AUTHOR CONTRIBUTIONS}

Conceptualization, RA and HM. Methodology, AS, and HM. Formal analysis, AS and HM. Investigation, AS and HM. Resources, HM. Data curation, AS. Writing-original draft preparation, RA. Writing-review and editing, RA and HM. Visualization, RA, AS, and HM. Supervision, HM. Project administration, HM. All authors contributed to the article and approved the submitted version.

\section{FUNDING}

This research was funded by National Institutes of Health, grant number P30 EY 026877, the National Center for Advancing Translational Sciences, National Institutes of Health, through Grant UL1TR002003 and a Research to Prevent Blindness unrestricted grant to Stanford Department of Ophthalmology.

Belief Differences. Am J Respir Crit Care Med (1998) 157(6):1810-7. doi: 10.1164/ajrccm.157.6.9712007

10. Ciechanowski, P, Russo, J, Katon, W, Simon, G, Ludman, E, Von Korff, M, et al. Where is the Patient? The Association of Psychosocial Factors and Missed Primary Care Appointments in Patients With Diabetes. Gen Hosp Psychiatry (2006) 28(1):9-17. doi: 10.1016/j.genhosppsych.2005.07.004

11. Mclean, S, Gee, M, Booth, A, Salway, S, Nancarrow, S, Cobb, M, et al. Targeting the Use of Reminders and Notifications for Uptake by Populations (TURNUP): A Systematic Review and Evidence Synthesis. Heal Serv Deliv Res (2014) 2(34):1-216. doi: 10.3310/hsdr02340

12. Akter, S, Doran, F, Avila, C, and Nancarrow, S. A Qualitative Study of Staff Perspectives of Patient Non-Attendance in a Regional Primary Healthcare Setting. Australas Med J (2014) 7(5):218-26. doi: 10.4066/AMJ.2014.2056

13. Rees, G, Leong, O, Crowston, JG, and Lamoureux, EL. Intentional and Unintentional Nonadherence to Ocular Hypotensive Treatment in Patients With Glaucoma. OPHTHA (2010) 117(5):903-8. doi: 10.1016/j.ophtha. 2009.10.038

14. Rees, G, Chong, XL, Cheung, CY, Aung, T, Friedman, DS, Crowston, JG, et al. Beliefs and Adherence to Glaucoma Treatment: A Comparison of Patients From Diverse Cultures. J Glaucoma (2014) 23(5):293-8. doi: 10.1097/ IJG.0b013e3182741f1c

15. Thaller, M, Tsermoulas, G, Sun, R, Mollan, SP, and Sinclair, AJ. Negative Impact of COVID-19 Lockdown on Papilloedema and Idiopathic Intracranial Hypertension. J Neurol Neurosurg Psychiatry BMJ Publishing Group (2020) 92 (7):795-7. doi: 10.1136/jnnp-2020-325519

16. Schectman, JM, Schorling, JB, and Voss, JD. Appointment Adherence and Disparities in Outcomes Among Patients With Diabetes. J Gen Intern Med (2008) 23(10):1685-7. doi: 10.1007/s11606-008-0747-1

17. Mason, C. Non-Attendance at Out-Patient Clinics: A Case Study. J Adv Nurs (1992) 17(5):554-60. doi: 10.1111/j.1365-2648.1992.tb02832.x 
18. Paterson, BL, Charlton, $\mathrm{P}$, and Richard, S. Non-Attendance in Chronic Disease Clinics: A Matter of Non-Compliance? J Nurs Healthc Chronic Illn (2010) 2(1):63-74. doi: 10.1111/j.1752-9824.2010.01048.x

19. Pesata, V, Pallija, G, and Webb, AA. A Descriptive Study of Missed Appointments: Families' Perceptions of Barriers to Care. J Pediatr Heal Care (1999) 13(4):178-82. doi: 10.1016/S0891-5245(99)90037-8

20. Macharia, WM, Leon, G, Rowe, BH, Stephenson, BJ, and Haynes, RB. An Overview of Interventions to Improve Compliance With Appointment Keeping for Medical Services. JAMA J Am Med Assoc (1992) 267(13):18137. doi: 10.1001/jama.1992.03480130129038

21. Hixon, AL, Chapman, RW, and Nuovo, J. Failure to Keep Clinic Appointments: Implications for Residency Education and Productivity. Fam Med (1999) 31(9):627-30.

Author Disclaimer: The content is solely the responsibility of the authors and does not necessarily represent the official views of the National Institutes of Health.
Conflict of Interest: The authors declare that the research was conducted in the absence of any commercial or financial relationships that could be construed as a potential conflict of interest.

Publisher's Note: All claims expressed in this article are solely those of the authors and do not necessarily represent those of their affiliated organizations, or those of the publisher, the editors and the reviewers. Any product that may be evaluated in this article, or claim that may be made by its manufacturer, is not guaranteed or endorsed by the publisher.

Copyright (c) 2021 Aziz, Shah and Moss. This is an open-access article distributed under the terms of the Creative Commons Attribution License (CC BY). The use, distribution or reproduction in other forums is permitted, provided the original author(s) and the copyright owner(s) are credited and that the original publication in this journal is cited, in accordance with accepted academic practice. No use, distribution or reproduction is permitted which does not comply with these terms. 